\title{
Transformation of Optical Anisotropy Origins in Perovskite-related Materials: A First-Principles Study
}

\author{
Tinghao Tong, ${ }^{\dagger}$ Ming-Hsien Lee,$\star$ Jun Zhang $*, \dagger$ \\ †School of Physical Science and Technology, Physical and Chemical Detecting Center, Xinjiang \\ University, 666 Shengli Road, Urumqi 830046, China \\ Department of Physics, Tamkang University, New Taipei 25137, Taiwan
}

*Corresponding Author.

E-mail addresses: zhangjunxju@163.com (J. Zhang) 


\section{Table of Contents}

Figure S1. Distorted $\left[\mathrm{TiO}_{6}\right]$ octahedral units in $\mathrm{BTO}(\mathrm{a})$, structures of $\mathrm{CsPbI}_{3}(\mathrm{~b})$ and $\mathrm{ClOK}_{3}$ (c).

Figure S2. Band structures and PDOS of $\mathrm{SMMO}_{\text {(a), }} \mathrm{CsPbI}_{3}(\mathrm{~b}), \mathrm{MAPbI}_{3}$ (c), and $\mathrm{ClOK}_{3}(\mathrm{~d})$.

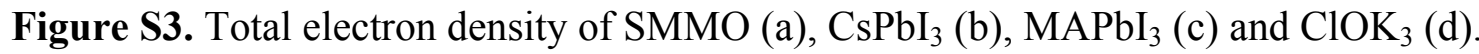

Figure S4. Total electron density planes of $\left[\mathrm{BO}_{3}\right],{ }^{1}\left[\mathrm{BO}_{4}\right]$ and ${ }^{2}\left[\mathrm{BO}_{4}\right]$ in $\mathrm{KBOC}$ lattice.

Table S1. Cut ionic radius for $\mathrm{MAPbI}_{3}, \mathrm{ClOK}_{3}$, and $\mathrm{KBOC}$ in real-space atom-cutting method.

Table S2. Response electron distribution anisotropy (REDA) contribution $(\Delta \rho)$ of measured units and real-space atom-cutting (RSAC) results $(\Delta \mathrm{n})$ of left units after atom-cutting. 
(a)

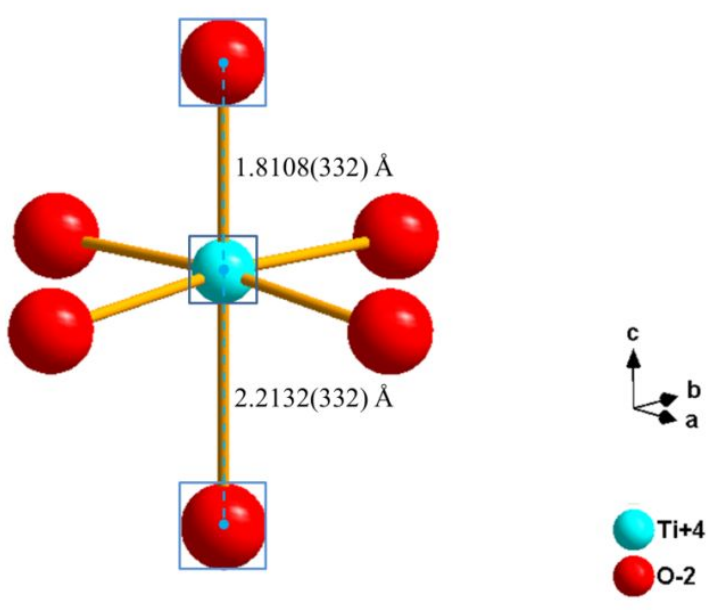

(b)

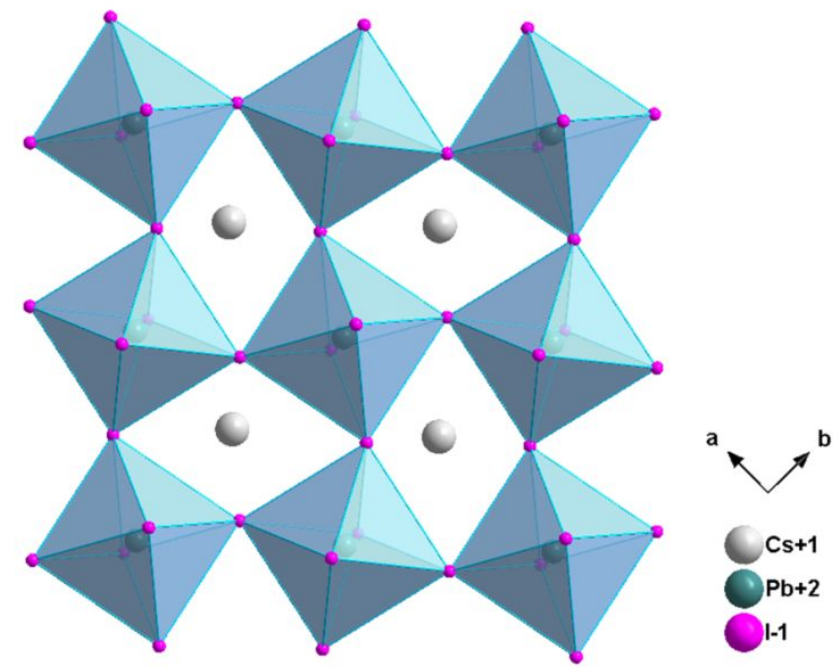

(c)

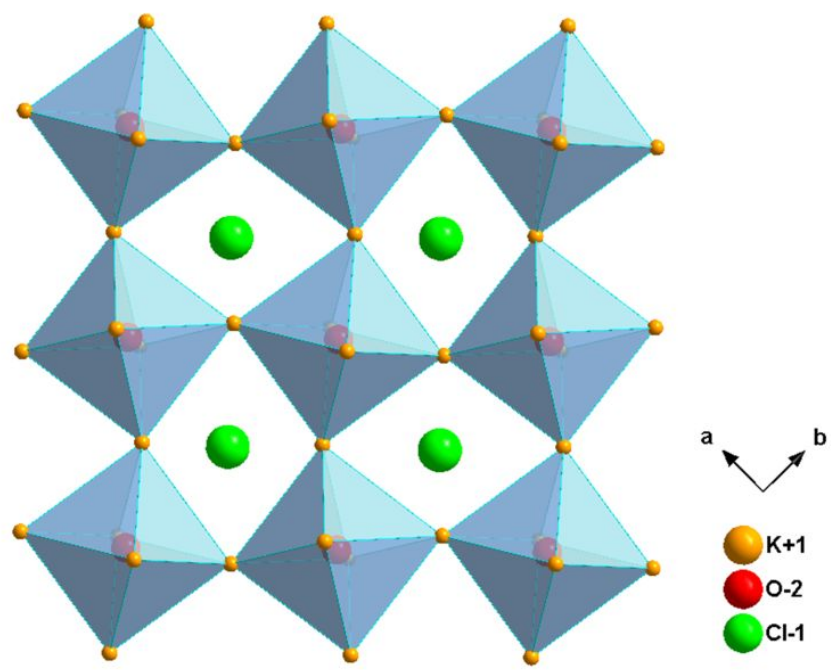

Figure S1. Distorted $\left[\mathrm{TiO}_{6}\right]$ octahedral units in $\mathrm{BTO}(\mathrm{a})$, structures of $\mathrm{CsPbI}_{3}(\mathrm{~b})$ and $\mathrm{ClOK}_{3}(\mathrm{c})$. 
(a)

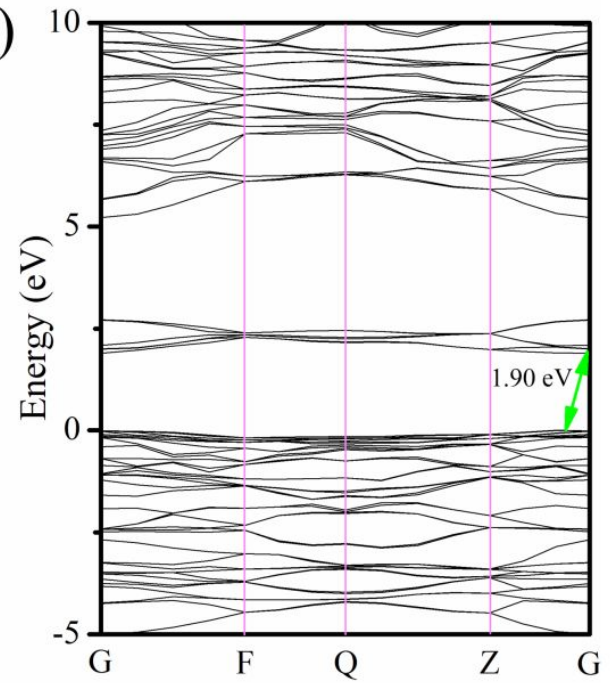

(b)

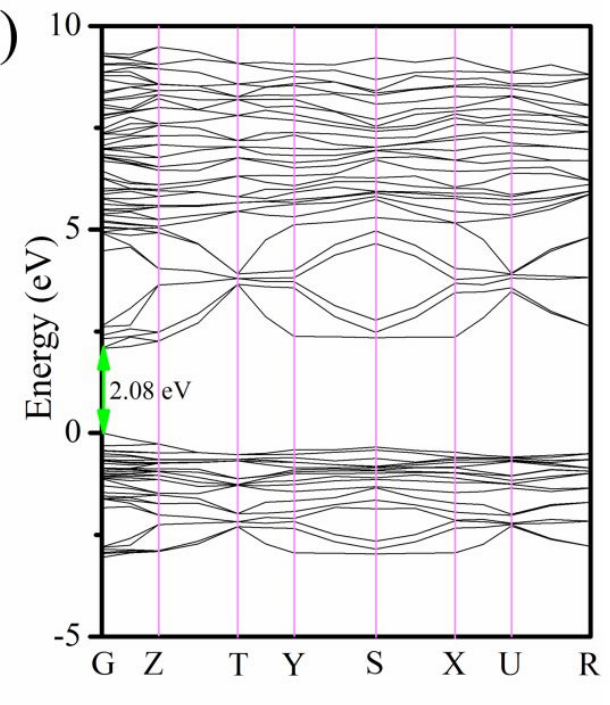

(c)

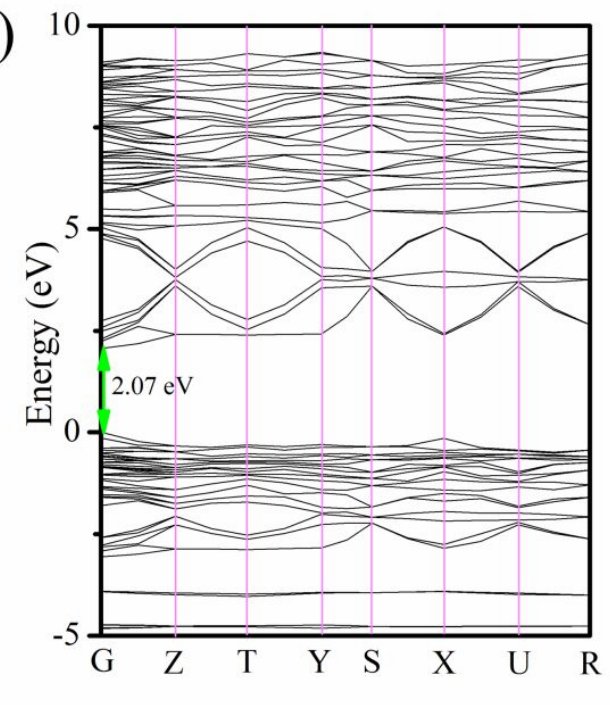

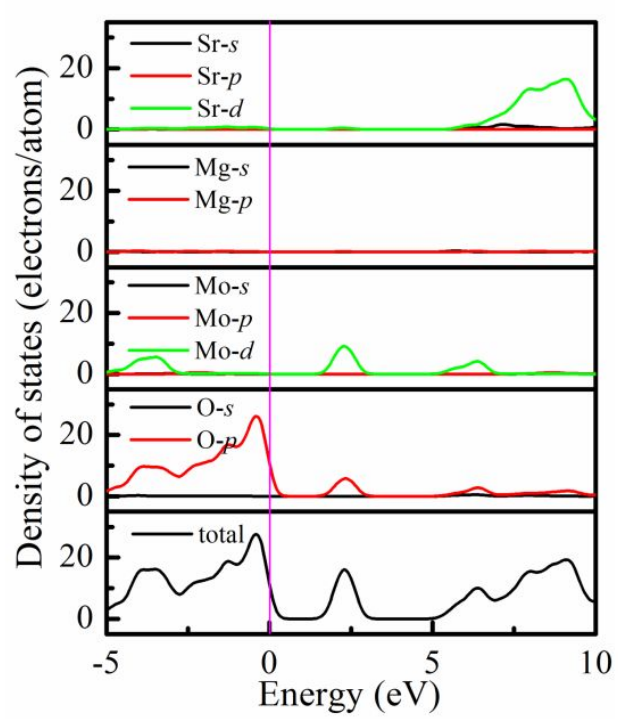
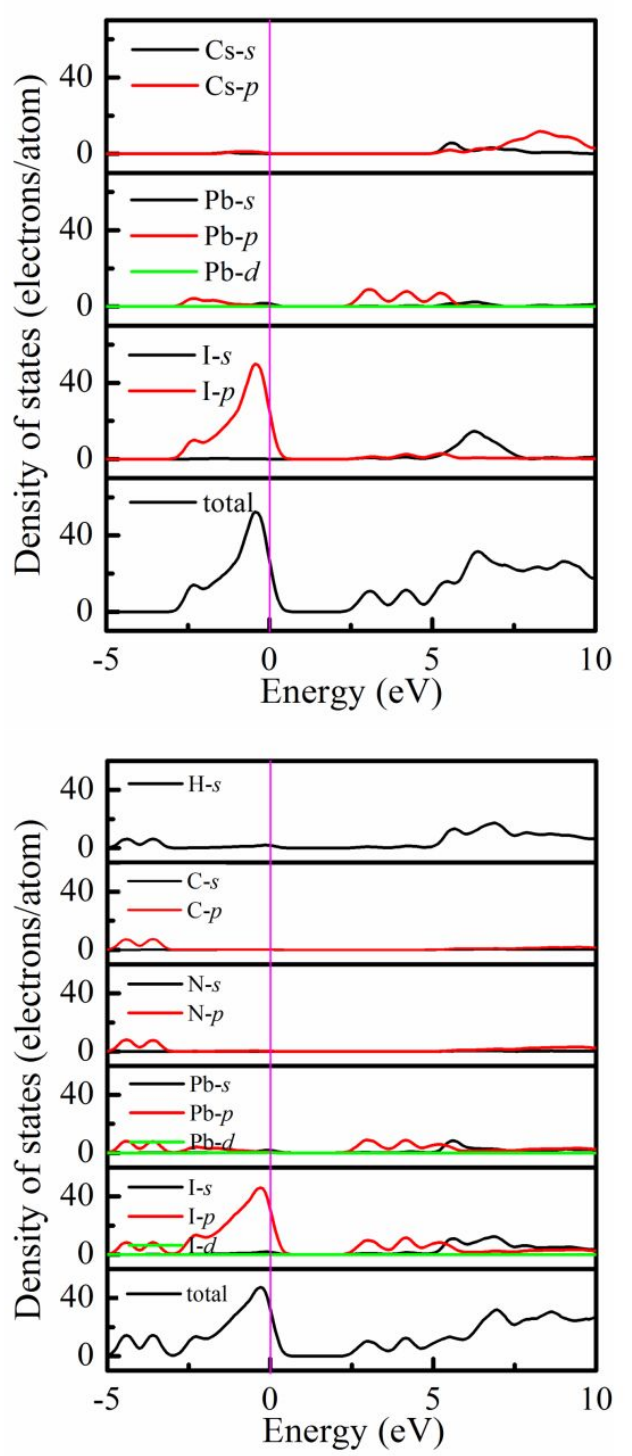
(d)
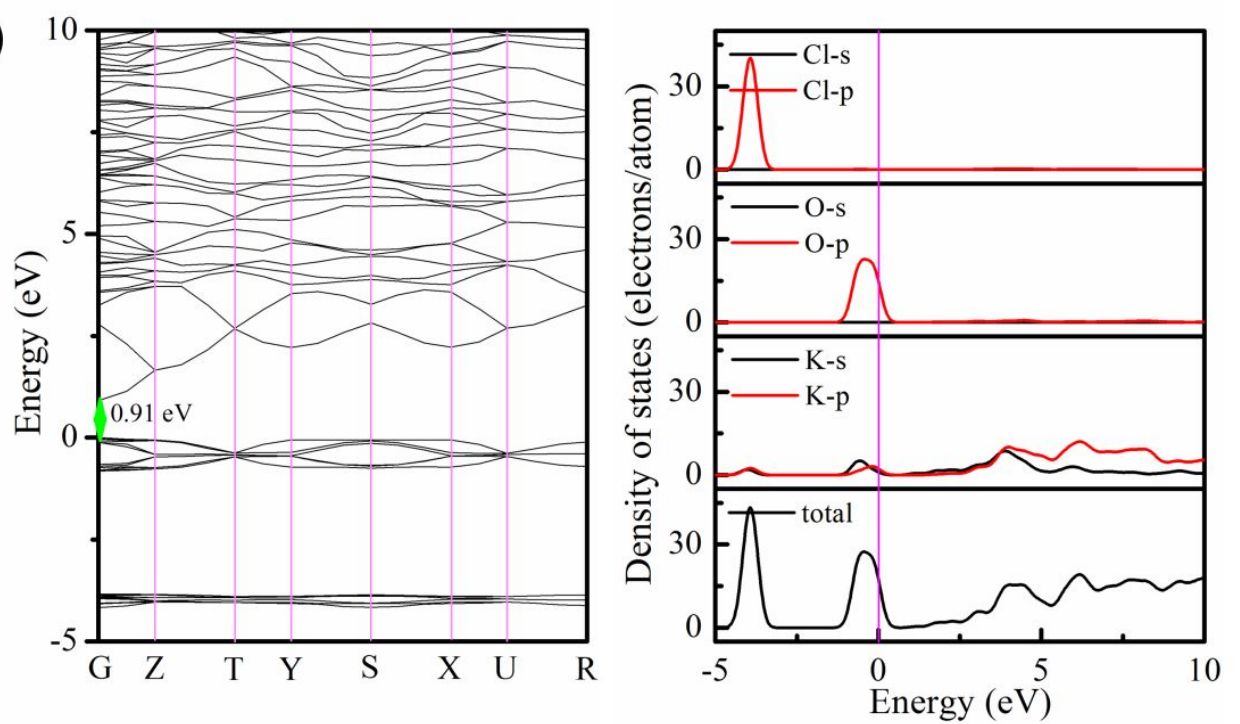

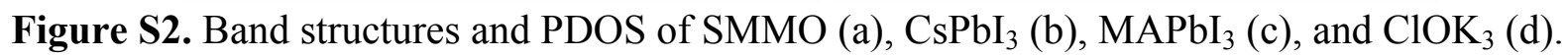

(a)

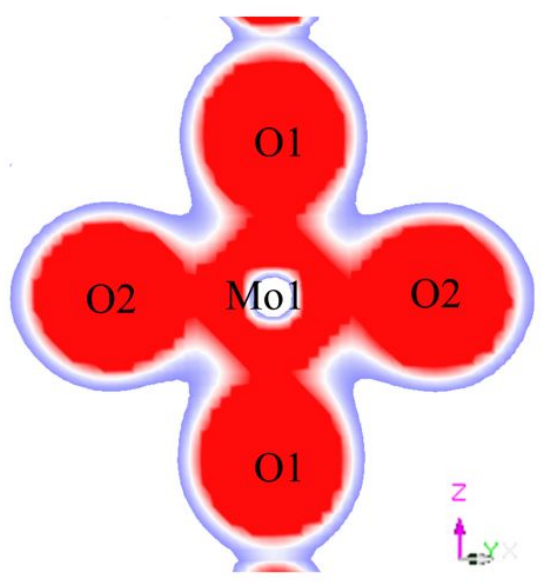

(b)

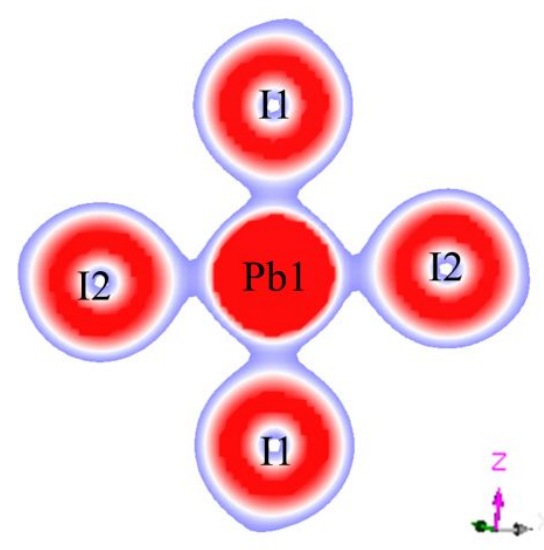

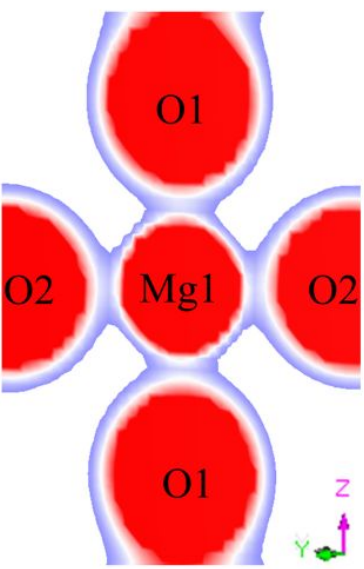

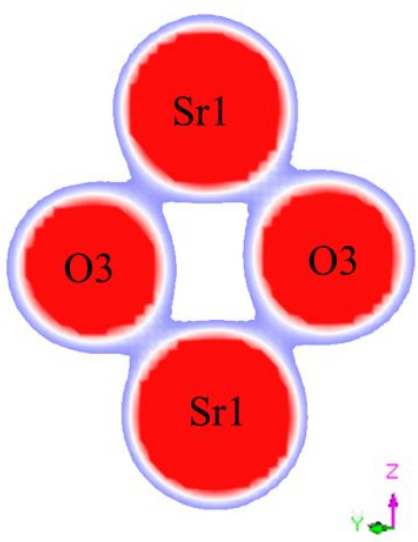

s
I2

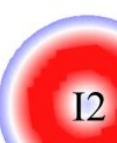

Cs1

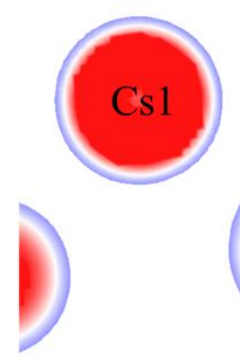

I2
Cs1

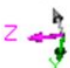


(c)

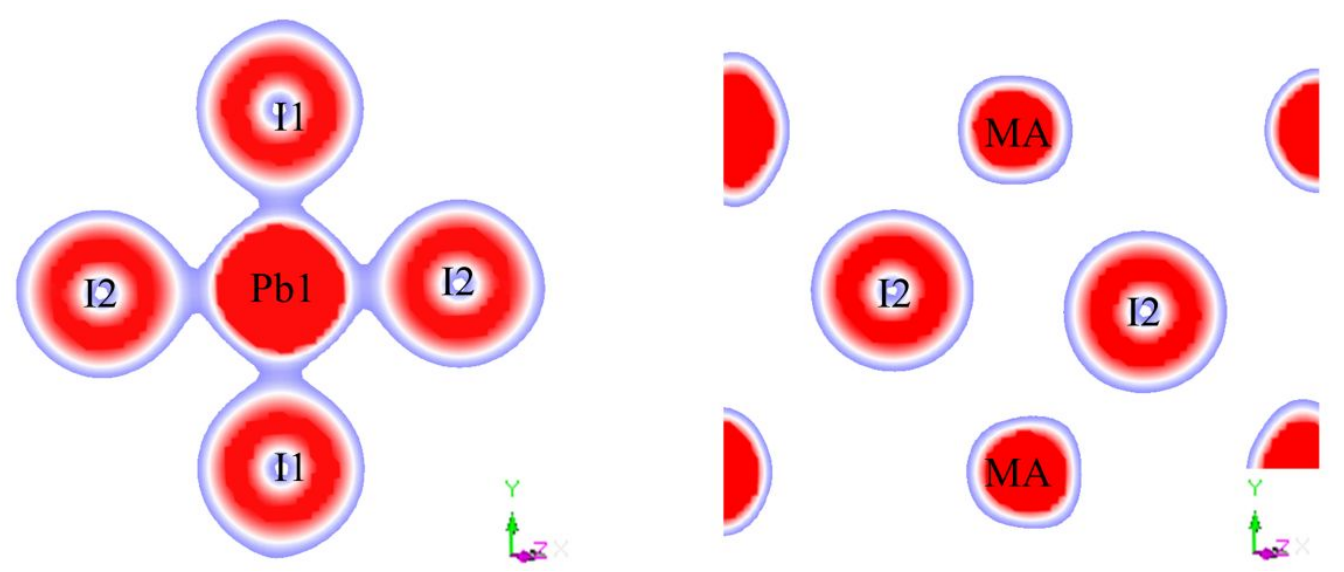

(d)

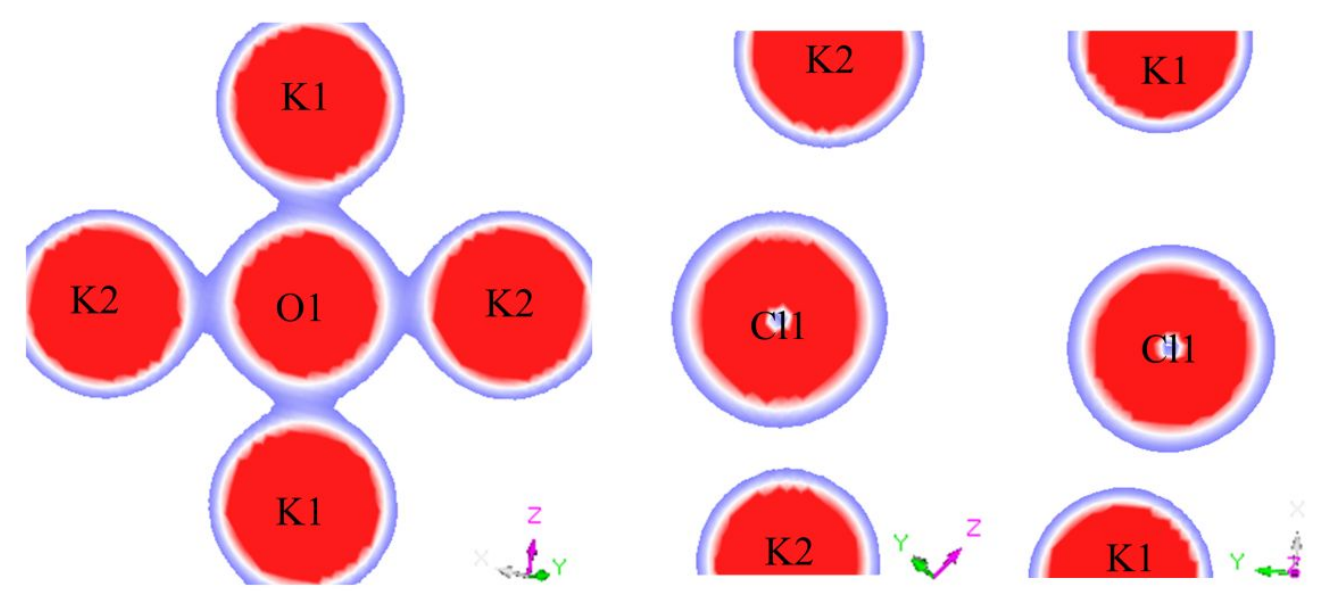

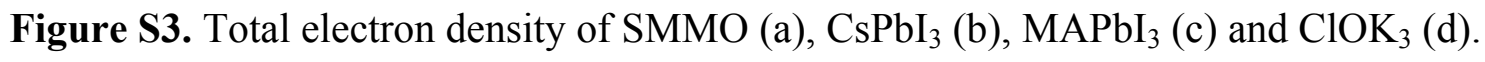

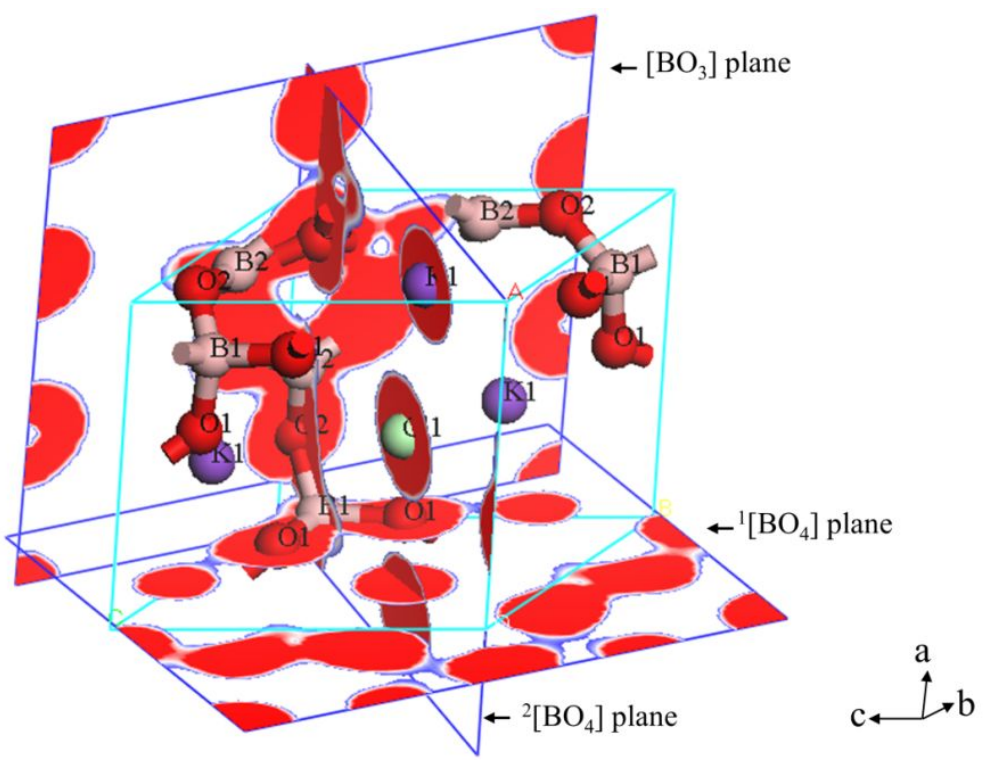

Figure S4. Total electron density planes of $\left[\mathrm{BO}_{3}\right],{ }^{1}\left[\mathrm{BO}_{4}\right]$ and ${ }^{2}\left[\mathrm{BO}_{4}\right]$ in $\mathrm{KBOC}$ lattice. 
Table S1. Cut ionic radius for $\mathrm{MAPbI}_{3}, \mathrm{ClOK}_{3}$, and $\mathrm{KBOC}$ in real-space atom-cutting method.

\begin{tabular}{cccc}
\hline Compound & Atom & Charge & Cut radius $(\AA)$ \\
\hline $\mathrm{MAPbI}_{3}$ & $\mathrm{~Pb}$ & +2 & 1.20 \\
\hline $\mathrm{ClOK}_{3}$ & $\mathrm{I}$ & -1 & 2.16 \\
$\mathrm{KBOC}$ & $\mathrm{Cl}$ & -1 & 1.81 \\
& $\mathrm{~K}$ & +1 & 1.33 \\
& $\mathrm{Cl}$ & -1 & 1.81 \\
\hline
\end{tabular}

Table S2. Response electron distribution anisotropy (REDA) contribution $(\Delta \rho)$ of measured units and real-space atom-cutting $(\mathrm{RSAC})$ results $(\Delta \mathrm{n})$ of left units after atom-cutting.

\begin{tabular}{|c|c|c|c|}
\hline Method & compounds & $\begin{array}{l}\text { Measured units (REDA) } \\
\text { /Left units (RSAC) }\end{array}$ & $\begin{array}{l}\Delta \rho(\mathrm{REDA}) \\
/ \Delta \mathrm{n}(\mathrm{RSAC})\end{array}$ \\
\hline \multirow{7}{*}{ REDA } & \multirow{2}{*}{ BTO } & {$\left[\mathrm{BaO}_{12}\right]$} & 0.000058 \\
\hline & & {$\left[\mathrm{TiO}_{6}\right]$} & 0.002585 \\
\hline & \multirow{3}{*}{ SMMO } & {$\left[\mathrm{SrO}_{12}\right]$} & 0.000202 \\
\hline & & {$\left[\mathrm{MoO}_{6}\right]$} & 0.001281 \\
\hline & & {$\left[\mathrm{MgO}_{6}\right]$} & -0.000248 \\
\hline & \multirow{2}{*}{$\mathrm{KBOC}$} & {$\left[\mathrm{B} 6 \mathrm{O}_{10}\right]$} & 0.011414 \\
\hline & & {$\left[\mathrm{ClK}_{6}\right]$} & 0.000482 \\
\hline \multirow{4}{*}{ RSAC } & $\mathrm{CsPbI}_{3}$ & {$\left[\mathrm{PbI}_{6}\right]$} & 0.291 \\
\hline & $\mathrm{MAPbI}_{3}$ & {$\left[\mathrm{PbI}_{6}\right]$} & 0.305 \\
\hline & $\mathrm{ClOK}_{3}$ & {$\left[\mathrm{OK}_{6}\right]$} & 0.043 \\
\hline & KBOC & {$\left[\mathrm{B}_{6} \mathrm{O}_{10}\right]$} & 0.045 \\
\hline
\end{tabular}

